\title{
Student perceptions of math-physics interactions throughout spins-first quantum mechanics
}

\author{
Benjamin P. Schermerhorn \\ ${ }^{1}$ Department of Physics and Astronomy, California Polytechnic University, Pomona, Pomona, CA, 91768, US \\ Armando Villasenor, ${ }^{1}$ Darwin Del Agunos, ${ }^{1}$ Homeyra Sadaghiani, ${ }^{1}$ Gina Passante, ${ }^{2}$ and Steve Pollock ${ }^{3}$ \\ ${ }^{2}$ Department of Physics and Astronomy, California State University, Fullerton, Fullerton, CA, 91768, US \\ ${ }^{3}$ Department of Physics and Astronomy, University of Colorado, Boulder, Boulder, CO, 80309, US
}

One of the purported benefits of teaching a spins-first approach to quantum mechanics is that it allows students to build up quantum mechanical ideas and learn postulates before moving to the more complicated mathematics used in the context of wave functions. In order to begin to explore this claim in a spins-first course, a survey was developed and administered as an extra credit activity at three different universities. All three universities teach spins-first quantum mechanics with interactive methods. This work compares students' responses to identical questions about the relationship between and difficulty of math and physics from two administrations of the survey given at the ends of the spins and wave functions portions of the course. Results offer insight into students' perspectives about the nature and difficulty of mathematics in these two paradigms of quantum mechanics. 


\section{INTRODUCTION}

Throughout a standard physics curriculum, students engage with various levels of mathematics, from basic calculus and vector products in introductory physics courses to vector calculus, differential equations, complex numbers, and linear algebra in the upper-division curriculum. This mathematics often provides the basis for understanding and exploring physical systems and as the physics systems become more complex in upper-division courses the mathematics also grows in sophistication. Quantum mechanics is no exception, as it introduces complex mathematical formalisms that students have not seen in prior courses. This course has often been shown to be very challenging for students solely in terms of the new, abstract concepts that are introduced by the course [1-6].

In the past decade, there have been two common paradigms of quantum instruction: spins-first (SF) and position-first (PF). Depending on the approach, the complexity and role of mathematics are drastically different in the beginning of a standard course. A SF approach introduces the postulates of quantum mechanics using a series of Stern-Gerlach experiments and discrete spin-1/2 bases. Grounded in Dirac notation, quantum states in a SF paradigm are represented as two-state vectors relating to measuring a particle to have spin up or spin down. After indepth discussions of quantum mechanical principles in this context, a transition is made to continuous bases and wave functions for a particle in various potential wells. As part of a spins-first course, the mathematics of linear algebra provides an accessible mathematical language in which students can grasp the abstract concepts and distinctly different way of thinking that appears in quantum mechanics before transitioning to the complex mathematical formalisms in a wave functions context.

A PF approach begins with the time-independent Schrodinger equation and continuous wave functions associated with measuring probabilities for the position, momentum, or energy of a particle. Students solve the Schrodinger equation (a differential equation of varying difficulty depending on the potential energy and the spatial dimensionality involved) to find the wave function, then use integration as a means to find probabilities or expectation values. In this approach, students are simultaneously introduced to the new ideas of quantum mechanics and a high level of mathematical rigor. Sadaghiani [1] argues that this approach gives more emphasis to mathematical skill solving complex integrals and differential equations than to the postulates of quantum mechanics.. Further, Johansson found that students in a PF course, either accepted having to learn quantum mechanics in this mathematical way or would experience "crisis" leading to the student distancing themselves from the subject of physics [7].

This paper presents the results of a preliminary survey given to students at different points in a SF course to identify if there were any differences in students' perspectives of the relationship between math and physics when they studied discrete spin systems and continuous wave functions of position. We further sought to investigate if the shift from discrete to continuous quantum mechanical systems resulted in a shift in students' self-perceptions about the challenges of mathematics and physics. A shift from seeing the physics as the more challenging aspect of the course when working with spin systems to the mathematics as the challenging component would support the idea that a spins-first approach allows students to develop the ideas of quantum mechanics within a simpler mathematical setting before transitioning to the more complex mathematics of continuous wave functions.

\section{METHODOLOGY}

In order to explore perspectives, the survey was administered to students at three different institutions all teaching a spins first approach to quantum mechanics. University $\mathrm{A}$, is a large, public, selective, $\mathrm{R} 1, \mathrm{PhD}$ granting institution. Universities B and C are large, public, Hispanicserving, primarily undergraduate institutions. Instructors at all three universities teach using a SF instructional paradigm using the same textbook, near identical, interactive instructional materials, and place emphasis on conceptual understanding of content. Both courses at Universities B and $\mathrm{C}$ follow a similar pace, but had only covered the mathematics of the infinite and finite square well potentials by the end of the semester. Additional topics covered at University A include the free particle, Hydrogen atom, harmonic oscillator, and angular momentum.

This paper focuses on two questions of a longer survey. The first question asks students to compare the relative challenge of the mathematics and physics concepts, while the second asks students to respond on a scale about the extent to which the mathematics or physics aids the understanding of the other. The questions are reproduced below in an abbreviated form.

1. Compare the role of math and physics concepts. Is the math more challenging than the concepts, or vice versa? Explain your response.

2. On a 5pt scale of strongly agree to strongly disagree, respond to the following two statements. Please elaborate on your responses.

a. Understanding the mathematics helped me comprehend the physics concepts.

b. Understanding the physics concepts helped me comprehend the mathematics.

The survey was administered online as an optional extracredit activity. Since the kind of mathematics used in the spins-context varies considerably to the mathematics in the wave functions context, the survey was given at multiple instances during the semester. Language was added at the beginning of each question to specifically focus the 
response to the current content of the course (e.g., "Considering the spins-portion of the course..."). The response rates for University A $(\mathrm{N}=69)$, University $\mathrm{B}$ $(\mathrm{N}=34)$ and University $\mathrm{C}(\mathrm{N}=37)$ on the spins version of the survey given during the middle of the semester were $70 \%$, $38 \%$, and $51 \%$. Response rates on the wave functions version given at the end of the semester were $50 \%, 53 \%$, and $54 \%$. For each version of the survey, some students left the first question blank but still answered question two, meaning the number of students from each school may not be the same between questions. Students were given alphanumeric codes prior to coding which blinded both the university and students' identity.

Initial data analysis of the spins version survey categorized students responses to each question, providing a count of students for each possible response (e.g., "math is more challenging" or "physics is more challenging"). The original 5-point scale for each of the scaled questions was reduced to a 3-point scale to simply identify agreement, neutrality, or disagreement to each statement.

Students' written explanations were coded by common phrases or ideas mentioned by the student. The data was blinded and independently coded by three of the authors. Authors did not operationalize definitions for what was math and what was physics, but based codes on key phrases in student responses. A final list of codes was discussed until agreement was reached among all authors. The list of codes was then used to analyze the results of the wave function version, but was modified to account for the introduction of new content material. Responses were not included in analysis where it was not sufficiently clear whether students were referring to mathematics ideas or physics concepts being more challenging.

\section{RESULTS}

From analysis of student responses to each of the two questions, we identify student perceptions of the relative difficulty of math and physics as well as the extent to which students feel each subject contribute the understanding of the other. Since data was collected from courses using the SF paradigm, the spins version of the survey given about halfway into the course refers to newly introduced quantum mechanics in the context of the Stern-Gerlach experiment and the associated linear algebra used to model the quantum systems. We compare the results to questions on this survey to another survey given at the end of the course referring to the second portion of the course where students work with wave functions and the Schrodinger equation.

\section{A. Analyzing the relative difficulty of math and physics}

When asked whether they found the math more challenging than physics concepts in the spins-portion of the course, $75 \%$ of students wrote that physics concepts were more difficult (Table 1). Exploring student explanations to the question, we categorized responses in terms of whether students felt that physics was more difficult or that the math was more difficult, since only 3 students answered the challenge was equal. There were four common reasons (stated by more than $10 \%$ of students) students identified for why physics was more difficult. Most often they would state that the math was familiar or simple $(40 \%)$, referring to different aspects of linear algebra or stating that that matrix calculation was fairly straightforward.

The math is pretty straightforward, it is for the most part just algebra with different notation (C22)

Other students felt that the physics was more challenging because the concepts required deeper understanding than the mathematics (29\%). $17 \%$ of students identified the difficulty because quantum mechanics was different than classical mechanics whereas another $17 \%$ specifically mentioned the physics was strange or they could not rely on previous physics intuition when trying to learn quantum mechanics.

Before [quantum] I was able to rely on my intuition to help me through physics, but it is no longer as applicable in this course. (A21)

The few students that responded that the math was more difficult in the spins portion of the course most commonly felt that the Dirac notation was difficult (38\%).

The most difficult concept in the course for me so far has been learning new forms of notation/totally new frameworks- such as braket notation. (A13)

Student responses here also showed that some students were unsure when to apply mathematics formalism or calculation to a physical situation (38\%).

Analysis of the second survey (given after the wave functions portion of the course) shows a shift in students responses at University A and University B to finding the mathematics as more challenging than the physics concepts (Table 2). In total, 53\% of students found the mathematics more challenging, compared to $38 \%$ of students finding the physics concepts as the more challenging aspect. This aligns with the idea that the physics concepts are easier for students at this time due to the time spent with spins.

Table I: Number of student answering whether the math or physics is more challenging in the spins-context.

\begin{tabular}{|c|cccc|}
\hline University & Physics & Math & Equal & N/A \\
\hline A & 23 & 6 & 3 & 1 \\
B & 10 & 1 & 1 & 1 \\
C & 9 & 1 & 0 & 0 \\
Total (\%) & $42(75 \%)$ & $8(14 \%)$ & $4(7 \%)$ & $2(4 \%)$ \\
\hline
\end{tabular}


Table II: Number of student answering whether they find the math or physics more challenging in the wave function context.

\begin{tabular}{|c|cccc|}
\hline University & Physics & Math & Equal & N/A \\
\hline A & 7 & 14 & 0 & 1 \\
B & 4 & 9 & 1 & 0 \\
C & 6 & 1 & 1 & 1 \\
Total (\%) & $17(38 \%)$ & $24(53 \%)$ & $2(4 \%)$ & $2(4 \%)$ \\
\hline
\end{tabular}

Few students, of those who stated that the mathematics was more challenging, stated they had a weaker mathematics background or had forgotten calculus (13\%).

\section{I had forgotten a lot of the calculus and Differential equation math that goes behind solving wave equations. (A47)}

Two students responded equal difficulty - both noted that they felt that the mathematics itself was tedious. $21 \%$ of students responded that they felt the spins portion of the class had given them a good foundation in the physics

\section{...math is more challenging because I already} understand the concepts from earlier on in the semester. not much has changed there (A61)

These responses support the idea that a spins-first curriculum allows students to build a conceptual understanding of quantum mechanical ideas before presenting the material with more challenging mathematics.

Many fewer students on the wave functions version of the survey felt that the physics was still more challenging than the mathematics. Results at school $\mathrm{C}$ remained consistent with students finding the physics still being more challenging. Students across all schools that responded in this way expressed comfort and mastery with calculus (35 $\%$ ), so therefore the mathematics was easier, or that the physics concepts still required deeper understanding (18\%).

The physics seems a bit more difficult than the math since the math is simply doing integrals. The actual effect on the physics is what I'm unsure of (C6).

Student responses show they initially struggle with physics concepts while finding the mathematics to be relatively easy and accessible. After the shift in context to wave functions, students share that they find the mathematics to be difficult, but that the spins-first approach alleviates some of the difficulty of the physics at this stage.

\section{B. Analyzing perceptions of the interconnectedness of math and physics}

When asked to mark whether mathematics helped physics and vice versa, most students commonly agreed with both statements: $48 \%$ on the spins version and $44 \%$ on the wave functions version. Tables III and IV show a breakdown of student responses for each survey. Despite the shift in students' perception of which area was more challenging, their responses stay mostly consistent in terms of the subject's interconnectedness. The only notable change between student responses is the slight increase in a neutral response to "Understanding the physics concepts helped me comprehend the mathematics." This could be due to the increased difficulty of the mathematics or in the connecting of the mathematics and physics concepts.

Analysis of student responses when they were asked to elaborate on their answers could be organized into three categories. They were either representative of the two unidirectional options where physics helps the mathematics or mathematics helps the physics, or that highlighted how mathematics and physics helped each other. Of the 65 students responding to the written question, 28 (43\%) elaborated on their agreement to both statements commonly stating that the math and physics built on each other.

The two above both go hand in hand. if your get the concept then that really helps to conceptually check the math..., and knowing what the math means can help you conceptually view the problems. (A57)

27 (42\%) students elaborated on how mathematics helped with physics.

I think the quantum mechanical concepts are too abstract for me to understand... I personally feel more comfortable with my math, so it's easier for me to make sense of the unfamiliar physical concepts using the familiar math I already understand. (B06)

Table III: Student responses to the mid-semester survey on the spins portion of the course

\begin{tabular}{|l|c|c|c|c|c|}
\hline \multicolumn{5}{|c|}{ The physics helps me make sense } \\
of the math \\
\cline { 2 - 7 } $\begin{array}{l}\text { The math } \\
\text { helps me } \\
\text { make sense } \\
\text { of the } \\
\text { physics }\end{array}$ & Agree (+) & 39 & 7 & 9 & $\mathbf{5 5}$ \\
\cline { 2 - 7 } & Neutral (0) & 6 & 3 & 2 & $\mathbf{1 1}$ \\
\cline { 2 - 7 } & Disagree (-) & 5 & 4 & 6 & $\mathbf{1 5}$ \\
\cline { 2 - 7 } & Total & $\mathbf{5 0}$ & $\mathbf{1 4}$ & $\mathbf{1 7}$ & $\mathbf{1 0 0 \%}$ \\
\hline
\end{tabular}


Table IV: Student responses on the end of semester survey on the wave functions portion of the course.

\begin{tabular}{|l|c|c|c|c|c|}
\hline \multicolumn{6}{|c|}{ The physics helps me make sense } \\
of the math
\end{tabular}

Only $10(15 \%)$ discussed how physics helped with the mathematics. The larger number of responses towards math helping physics and the math and physics helping each other, compliments earlier findings that students find the mathematics easier and acknowledge that it helps them make sense of the quantum mechanical topics.

47 students on the wave functions version of the survey answered the elaboration question. Responses here still favored math and physics helping each other $(19 / 40 \%)$ and Mathematics helping physics $(16 / 34 \%)$, but were more equally spread as $12 / 26 \%$ students still elaborated on physics helping with understanding the mathematics. Subcategories of responses were replicated in the second survey, showing that students still felt the mathematics and physics are strongly connected throughout the course, as exemplified by the following quotes.

\section{I think they both go hand in hand. For understanding wave functions, some concepts are better understood with math and other with physics (C14)}

The physics makes the math easier to visualize and the math provides a base for physical intuition. For example, the properties of wave functions helped me understand Fourier transforms. (A52)

Students articulated the extent to which the mathematics and physics contributed to a complete understanding of quantum mechanics. In both the spins and wave functions contexts, students describe mathematics as assisting or even driving the learning of the physics. In turn, students articulated that physics made the mathematical processes easier to understand and built "a better understanding of or intuition for" the mathematical processes.

\section{DISCUSSIONS}

Preliminary student surveys given at two points during a spins-first (SF) course showed a shift in student perceptions of math and physics from finding quantum physics concepts more challenging during the spins portion of the course to finding the mathematics as the challenging aspect during the wave functions portion of the course. This is in line with the idea that SF approach to quantum mechanics allows students to learn new quantum mechanics concepts with simpler mathematical representations before moving to the more abstracted continuous systems. Student written responses further support this claim, where students felt that they had a strong physics foundation.

Despite the shifting perspectives, at both points in the semesters a large majority of students responded that understanding math helped with understanding physics and vice versa. Student written comments highlighted perceived connections between mathematics and physics. At both points in the semester, the majority of students responded along the lines that mathematics and physics at this level were "interconnected" or went "hand-in-hand." In the spinsversion of the survey a near majority of students exclusively provided statements of mathematics helping with their understanding of physics, while very few students wrote solely about cases where physics helped them understand the mathematics. In the final survey the responses were more evenly distributed between the three categories. This is likely due to the fact that the quantum mechanical concepts had been established by this point in the semester but the mathematics context was no seen as more challenging.

While Johansson [7] found that students would either have to accept the difficulty of the mathematics or become alienated by quantum mechanics, these results suggest that the SF paradigm may allow students to enter quantum mechanics without immediately experiencing difficulty with the mathematics. Ongoing work seeks to address these questions further in both SF and PF classrooms in order to make a direct comparison of student perceptions of the mathematics and physics content within the two paradigms.

\section{ACKNOWLEDGMENTS}

This work has been supported in part by the NSF under Grants No. DUE-1626594, 1626280, and 1626482.
[1] H. R. Sadaghiani, in 2016 Phys. Educ. Res. Conf. Proc. (Sacramento, CA, 2016).

[2] C. Singh and E. Marshman, Phys. Rev. Spec. Top. - Phys. Educ. Res. 11, 020117 (2015).

[3] E. Gire and E. Price, Phys. Rev. Spec. Top. - Phys. Educ. Res. 11, 020109 (2015).
[4] B. W. Dreyfus, E. R. Sohr, A. Gupta, and A. Elby, in 2015 Phys. Educ. Res. Conf. Proc. (2015), pp. 111-114.

[5] H. Sadaghiani and L. Bao, in 2005 Phys. Educ. Res. Conf. Proc. (2006), pp. 61-65.

[6] C. Singh, Am. J. Phys. 69, 885 (2001).

[7] A. Johansson, Eur. J. Phys. 39, 025705 (2018). 